\title{
Role of Solvent for the Dynamics and the Glass Transition of Proteins
}

\author{
Helén Jansson, Rikard Bergman, and Jan Swenson* \\ Department of Applied Physics, Chalmers University of Technology, SE-412 96 Göteborg, Sweden
}

ABSTRACT: For the first time, a systematic investigation of the glass transition and its related dynamics of myoglobin in waterglycerol solvent mixtures of different water contents is presented. By a combination of broadband dielectric spectroscopy and differential scanning calorimetry (DSC), we have studied the relation between the protein and solvent dynamics with the aim to better understand the calorimetric glass transition, $T_{\mathrm{g}}$, of proteins and the role of solvent for protein dynamics. The results show that both the viscosity related $\alpha$-relaxation in the solvent as well as several different protein relaxations are involved in the calorimetric glass transition, and that the
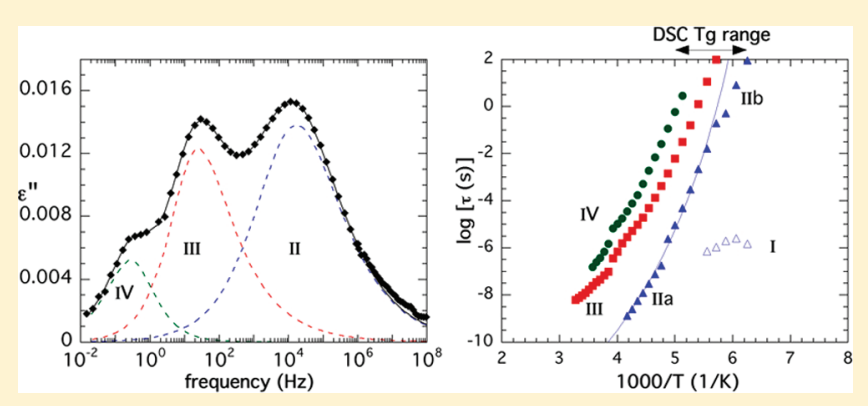
broadness $\left(\Delta T_{\mathrm{g}}\right)$ of the transition depends strongly on the total amount of solvent. The main reason for this seems to be that the protein relaxation processes become more separated in time with decreasing solvent level. The results are compared to that of hydrated myoglobin where the hydration water does not give any direct contribution to the calorimetric $T_{\mathrm{g}}$. However, the large-scale $\alpha$-like relaxation in the hydration water is still responsible for the protein dynamics that freeze-in at $T_{\mathrm{g}}$. Finally, the dielectric data show clearly that the protein relaxation processes exhibit similar temperature dependences as the $\alpha$-relaxation in the solvent, as suggested for solvent-slaved protein motions.

\section{INTRODUCTION}

Proteins cannot function without surrounding hydration water. It has, for instance, been shown that the enzymatic activity of the model protein lysozyme is absent in the dehydrated state up to about $0.2 \mathrm{~g}$ of water/g of protein, and that roughly an equal fraction of water and protein is required for its full activity. 1,2 Similar behavior also appears to be typical for other globular proteins. ${ }^{1}$ The reason for this is that the function of a protein involves conformational changes of the protein structure, which, in some way, are promoted by the solvent. However, exactly how the solvent enables protein structure fluctuations is still a matter of discussion. $^{3-8}$

The dynamics of a protein is determined by its energy landscape, ${ }^{9}$ but due to the complex structure of a protein, this energy landscape is basically impossible to determine. The situation is very similar to ordinary glasses (for proteins at low temperatures) and supercooled liquids (for proteins above the glass-transition-like onset of largescale motions around $200 \mathrm{~K}),{ }^{9,10}$ with the exception that for proteins the increase of fluctuations with increasing temperature is not directly caused by the increasing thermal energy. Rather, the protein motions are promoted by the temperature induced increasing motions in the solvent. ${ }^{6}$ Thus, the motion of a protein from one substate to another seems to be "driven" by the solvent. A molecular dynamics (MD) study by Tarek and Tobias ${ }^{5}$ suggested that translational motions in the solvent are required for substantial protein dynamics, whereas local rotations and reorientations of the solvent molecules have almost no influence on the protein dynamics. Similar conclusions were also drawn by Doster and Settles ${ }^{7}$ from studies where a combination of several techniques was used. It was suggested that it is the viscosity of the hydration water that is the essential coupling parameter between a protein and its surrounding solvent. A somewhat different idea was proposed by Frauenfelder and co-workers, ${ }^{4,8}$ who suggested that the $\beta$-relaxation in the hydration shell controls local ( $\beta$-like) fluctuations in the internal part of the protein and that the biologically most important largescale $(\alpha$-like $)$ fluctuations in the protein are determined by the cooperative and viscosity related $\alpha$-relaxation in the bulk solvent. Consequently, when the $\alpha$-relaxation of the solvent freezes in or disappears around the glass transition temperature $T_{g}$, also the more large-scale protein motions are expected to vanish.

The glass transition of hydrated proteins has been investigated by calorimetric and rheologic measurements (see, e.g., refs $11-15)$, and it has been shown that, except for the fact that this transition can be tricky to observe, it is exceptionally broad; ${ }^{12,14,15}$ i.e., the protein glass transition is not characterized by one specific temperature or a narrow temperature range. The broadness has been discussed in terms of a size distribution of water clusters on the protein surface ${ }^{15}$ or a large distribution of relaxation times, due to a large number of relaxing local regions, within the proteinwater system. ${ }^{12}$ Recently, it was, in fact, shown that a number of different protein relaxations participate in the glass transition region of hydrated proteins. ${ }^{16}$

For systematic investigations of the dynamics of proteins and its surrounding solvent, and how they are related to each other, it is useful to change both the total solvent content as well as the dynamical properties of the solvent. In the present study, this has

\footnotetext{
Received: September 20, 2010

Revised: February 23, 2011

Published: March 22, 2011
} 
been achieved by using different compositional mixtures of water and glycerol as a solvent. The advantage of using this type of mixture is fourfold:

(1) Biological function can be preserved even at high glycerol contents (up to at least $90 \mathrm{wt} \%$ glycerol in the case of, for instance, the globular protein lysozyme ${ }^{17}$ ).

(2) The addition of glycerol suppresses the probability of water to crystallize in the supercooled regime.

(3) The glycerol molecules are, like water, also forming hydrogen bonds to each other and to biological surfaces.

(4) The dynamical properties of the solvent can be substantially changed by changing the glycerol/water ratio.

The aim of the present calorimetric and dielectric study is to relate the calorimetric glass transition region $\Delta T_{\mathrm{g}}$ in the protein-solvent (water-glycerol) system to the relaxation processes obtained by dielectric spectroscopy. This in turn gives the opportunity to investigate the relation between protein and solvent dynamics, and how the relaxational behavior changes compared to that of hydrated proteins. From our results, it is clear that the calorimetric glass transition is in fact due to the freezing-in of both the $\alpha$-relaxation in the solvent as well as several protein relaxations occurring on different time scales. Furthermore, it is evident that the protein motions are directly related to the solvent dynamics. In the case of hydrated proteins, the hydration water undergoes a dynamic crossover when a local low-temperature water relaxation transforms to an $\alpha$-like relaxation at $160-180$ $\mathrm{K} .{ }^{18}$ This onset of more cooperative and long-range water motions at the crossover temperature seems to be responsible also for the glass-transition-like onset of large-scale protein fluctuations.

\section{EXPERIMENTAL SECTION}

The protein investigated in this study was freeze-dried horse heart myoglobin powder purchased from Sigma. The protein powder was mixed with certain amounts and compositions of water-glycerol mixtures to give 11 samples in total. The mixing procedure was performed by repeatedly adding small amounts of the freeze-dried protein powder to the mixture, and stirring. The different compositions, given in wt \% water and total solvent level $h$ ( $h=\mathrm{g}$ of solvent/g of protein), are as follows: $20 \mathrm{wt} \%(h=1$ and $h=2), 33 \mathrm{wt} \%(h=1$ and $h=2), 40 \mathrm{wt} \%(h=$ 1 and $h=2)$, and 50 wt $\%(h=1$ and $h=2)$. For reference, we also investigated three protein samples with single solvents, i.e., with 100 wt $\%$ glycerol $(h=1)$ and 100 wt $\%$ water (hydrated to $h=0.5$ and $h=0.33$ ), and all the corresponding pure solvent mixtures. The preparation of the hydrated proteins was performed in $100 \%$ humidity (in a desiccator). Finally, it should be noted that the basic water content of approximately 0.1 wt $\%$ was not taken into account in the hydration levels given above.

2.1. Differential Scanning Calorimetry. Differential scanning calorimetry (DSC) measurements were performed on TA Instruments DSC Q1000. For each measurement, the sample $(\approx 5-10 \mathrm{mg}$ ) was placed in a hermetic pan of aluminum, and as a reference, an empty hermetic pan was used. After equilibration at $95 \mathrm{~K}$, the measurement of each sample was carried out in the heating mode (heating rate $5 \mathrm{~K} / \mathrm{min}$ ) in the temperature interval 95-295 K. The temperature for the inflection point of the glass transition $\left(T_{\mathrm{g}}\right)$ was determined using the Universal Analysis 2000 software (TA Instruments), and the broadness of the glass transition by analyzing the derivative of the heat flow with respect to the temperature. Each measurement was carried out several times to ensure reproducibility of the data.

2.2. Dielectric Spectroscopy. The dielectric measurements were performed on a broadband dielectric spectrometer from Novocontrol. The samples were investigated in the frequency and temperature ranges $10^{-2}-10^{9} \mathrm{~Hz}$ and $120-350 \mathrm{~K}$, respectively. For the lower frequencies $\left(10^{-2}-10^{7} \mathrm{~Hz}\right)$, an Alfa-S High Resolution Dielectric Analyzer was used, and the measurements in the higher frequency range $\left(10^{6}-10^{9} \mathrm{~Hz}\right)$ were carried out using an Agilent 4291B RF Impedance Analyzer. For each measurement, the sample of thickness $0.1 \mathrm{~mm}$ (determined by silica spacers) was placed between two gold plated electrodes of 20 and $10 \mathrm{~mm}$ diameter for the low and high frequency measurements, respectively. In order to reduce the large contribution of conductivity and electrode polarization to the spectra at low frequencies, we also measured the samples with a Teflon film of $100 \mu \mathrm{m}$ thickness placed between the sample and one of the electrodes. This type of setup has been shown to reduce such effects. ${ }^{19,20}$ However, since the use of a Teflon layer may cause unwanted peaks ${ }^{21}$ in the data, the measured results were compared with some corresponding results obtained without the Teflon layer as well as measurements on the corresponding pure solvents with and without the same Teflon film. In addition, the results obtained with the Teflon setup were verified by analyzing the imaginary part of the permittivity with the dc-conductivity subtracted and the derivative of the real part of the permittivity ${ }^{22}$ for the samples without Teflon. However, although this careful analysis of the data obtained with the Teflon setup was needed, these measurements simplified the analysis of the relaxation process temperature dependences, which thereby motivated the use of the Teflon setup.

After preparation, each sample was placed in a sample holder and cooled down to $120 \mathrm{~K}$ and then reheated to $350 \mathrm{~K}$ while isothermal $( \pm 0.2 \mathrm{~K})$ scans were made at every fifth degree. The imaginary part of the dielectric response, $\varepsilon^{*}(f)=$ $\varepsilon^{\prime}(f)-\mathrm{i} \varepsilon^{\prime \prime}(f)$, was then analyzed. The dielectric loss peaks obtained for each sample were fitted by several Havriliak-Negami functions ${ }^{23}$ (eq 1) and one more general fit function $^{24}$ (eq 2)

$$
\begin{gathered}
\varepsilon^{\prime \prime}(\omega)=\sum \operatorname{Im}\left(\frac{\varepsilon_{\mathrm{s}}-\varepsilon_{\infty}}{\left(1+(\mathrm{i} \omega \tau)^{\alpha}\right)^{\beta}}\right) \\
\varepsilon^{\prime \prime}(\omega)=\frac{\varepsilon_{\mathrm{p}}^{\prime \prime}}{\frac{(1-C)}{a+b}\left[b\left(\omega / \omega_{\mathrm{p}}\right)^{-a}+a\left(\omega / \omega_{\mathrm{p}}\right)^{b}\right]+C}
\end{gathered}
$$

where $\omega=2 \pi f$ is the angular frequency. Specific parameters for the equations are in eq 1 the relaxation time $\tau$, the static dielectric constant $\varepsilon_{s}$, and the limiting value of the dielectric constant at high frequencies $\varepsilon_{\infty}$. The shape parameters $\alpha$ and $\beta$ determine the symmetric and asymmetric broadening of the relaxation peak, respectively. In eq $2, \omega_{\mathrm{p}}$ and $\varepsilon_{\mathrm{p}}^{\prime \prime}$ are the position and the height of the peak. The parameters $a$ and $b$ are shape parameters that describe, in a log-log plot, the slope of the peak at the low and high frequency side, respectively, and the $C$ parameter is a parameter that describes the broadening of the relaxation peak without changing the power laws at the high and low frequency sides.

The temperature dependences of the obtained relaxation processes were described by the Vogel-Fulcher-Tammann 
(VFT) equation ${ }^{25-27}$ (eq 3) or by the Arrhenius law (eq 4)

$$
\begin{gathered}
\tau=\tau_{0} \exp \left(\frac{D T_{0}}{T-T_{0}}\right) \\
\tau=\tau_{0} \exp \left(E_{\mathrm{a}} / k_{\mathrm{B}} T\right)
\end{gathered}
$$

where $\tau_{0}$ is the relaxation time extrapolated to infinite temperature, $E_{\mathrm{a}}$ is the activation energy, and $T_{0}$ is the temperature where $\tau$ would go to infinity. The parameter $D$ determines the deviation from Arrhenius temperature dependence. Equations 3 and 4 are generally used to describe the $\alpha$-and $\beta$-relaxations, which are due to global configurational changes and more local movements in the material, respectively.

\section{RESULTS}

In Figure 1, we show calorimetric glass transitions as obtained from DSC scans on myoglobin in a variety of mixtures of different water contents and solvent levels. For comparison, the result from one of the hydrated protein samples $(h=0.33)$ is also shown. The upper panel of Figure 1 shows the results for myoglobin in the mixture containing $20 \mathrm{wt} \%$ water at the solvent levels $h=1$ (A) and $h=2$ (B), and the middle panel the mixture of $33 \mathrm{wt} \%$ water at $h=1$ (C) and $h=2$ (D). The lower panel $(\mathrm{E})$ and $(\mathrm{F})$ shows the results for the mixture with $40 \mathrm{wt} \%$ water at $h=1$, and myoglobin hydrated to a level of $h=0.33$, respectively. From this figure and Figure $2 \mathrm{~A}$ and $\mathrm{B}$, it can be seen that the glass transition temperature $T_{\mathrm{g}}$ (given by the inflection point) as well as the glass transition range, $\Delta T_{\mathrm{g}}$ (taken from the onset to the end temperature of the transition), decreases with increasing water content for a specific solvent level. For instance, when the water content is increased from 20 to $40 \mathrm{wt} \%, T_{\mathrm{g}}$ decreases with $\approx 15 \mathrm{~K}$ independent of the total solvent level, and the glass transition range becomes somewhat narrower. It can also be seen that the glass transition range and the glass transition temperature decrease with increasing solvent level, suggesting that not only the time scale of the solvent dynamics is important for the protein dynamics but also the amount of solvent, as observed in a recent quasielastic neutron scattering study. ${ }^{28}$

In Figure 3, typical dielectric loss spectra are shown for three of the measured samples with Teflon film. From these figures, it is evident that several relaxation processes are present in the data, whereas contributions from $\mathrm{dc}$ conductivity and polarization effects are almost negligible due to the use of the Teflon film. Without the Teflon film, it is more difficult to determine the relaxation processes that are observed in the low frequency and high temperature range. It is important to note, however, that the processes can be observed also without Teflon if the derivative $^{22}$ of the real part of the permittivity is analyzed or the dc-conductivity is subtracted from the imaginary part of the permittivity, as shown in Figure 4. In this figure, the imaginary part of the permittivity of myoglobin in a water-glycerol solvent of 33 wt \% water $(h=1)$ is compared in measurements with and without the Teflon setup. It can be seen that, if the dcconductivity is subtracted from the data taken without using the Teflon setup, the resulting data are very similar to the data taken with the Teflon setup, although the peaks become slightly more pronounced when the Teflon setup is used. This similarity justifies our experimental approach to use the Teflon setup in our measurements.
All processes shown in Figure 3 were curve fitted using the Havriliak-Negami function (eq 1), except the slowest one for which eq 2 was used. In Figure 5, the temperature dependent relaxation times of the processes obtained from the curve fitting of the experimental data are shown together with the glass transition range determined by DSC. The figure shows two solvent processes, where the fastest one (process I) is a local water process that is commonly observed in systems of confined supercooled water. ${ }^{29}$ Its origin is not fully clear, but most likely it is due to reorientations of single water molecules. The second fastest process, denoted IIa or IIb depending on the temperature and sample composition, is the observed main relaxation of the solvent, in accordance with the observed dielectric relaxation processes of the corresponding bulk solvents. ${ }^{30,31}$ At higher temperatures, above the dynamic crossover temperature (see below), this process is denoted IIa and corresponds to the viscosity and glass transition related $\alpha$-relaxation in the solvent; i.e., in Figure $5 \mathrm{~A}-\mathrm{E}$, it is due to the $\alpha$-relaxation of the mixture, and in Figure 5F, to the relaxation of the hydration water. It should however be noted that even if process IIa is dominated by the relaxation of the solvent it also contains a smaller contribution of local protein motions on a similar time scale, as previously has been observed by both quasielastic neutron scattering (QENS) ${ }^{28,32}$ and time domain dielectric spectroscopy (TDDS). ${ }^{33}$

Below the crossover temperature, this relaxation (IIb) is due to a local $\beta$-relaxation of confined water molecules, ${ }^{15,18,29,34}$ or even more exactly, due to a secondary relaxation of JohariGoldstein type. ${ }^{35}$ It should here be noted that this low temperature water relaxation (IIb) is also commonly denoted w- or $v$-relaxation. ${ }^{35,36}$ Further evidence showing that this low temperature process is a water relaxation of local character has also been obtained from $1 \mathrm{D}$ and $2 \mathrm{D}^{2} \mathrm{H}$ NMR investigations. ${ }^{37,38}$ Thus, the reason for the dynamic crossover from process IIa to $\mathrm{IIb}$ is that at this temperature the local water relaxation becomes decoupled from the viscosity related structural $\alpha$-relaxation of the solvent. Below the crossover temperature, the $\alpha$-relaxation of the water-glycerol mixtures is generally weak and difficult to observe (or even nonobservable in the case of hydration water ${ }^{18}$ ). Therefore, its temperature dependence is extrapolated by the VFT fits shown in Figure 5.

In Figure 6, we show the composition dependence of the main solvent relaxation (process II) and the two fastest protein processes (III and IV), as well as a comparison with results found in the literature. In Figure 6A, it can be seen that the viscosity related process IIa becomes significantly faster with increasing water fraction for a given solvent content, and also slightly faster with increasing total solvent content. In addition to the solvent processes I and II, the samples exhibit also slower relaxation processes due to protein motions, as shown in Figure 5. The fastest of these processes (process III) is, based on earlier results from time-domain spectroscopy, ${ }^{39}$ attributed to be due to the relaxation of protein polar side groups, which are probably relaxing together with associated water molecules. Probably due to preferential hydration, ${ }^{40}$ the time scale of this process is only slightly dependent on the composition of the solvent, as shown in Figure 6B. This is also true for process IV shown in Figures 5 and 6C. However, it should be noted that the origin (which will be discussed below) of this process, as well as the remaining relaxation process of myoglobin in the mixture of $20 \mathrm{wt} \%$ water and $h=2$ (Figure 5B), is not fully clear. 

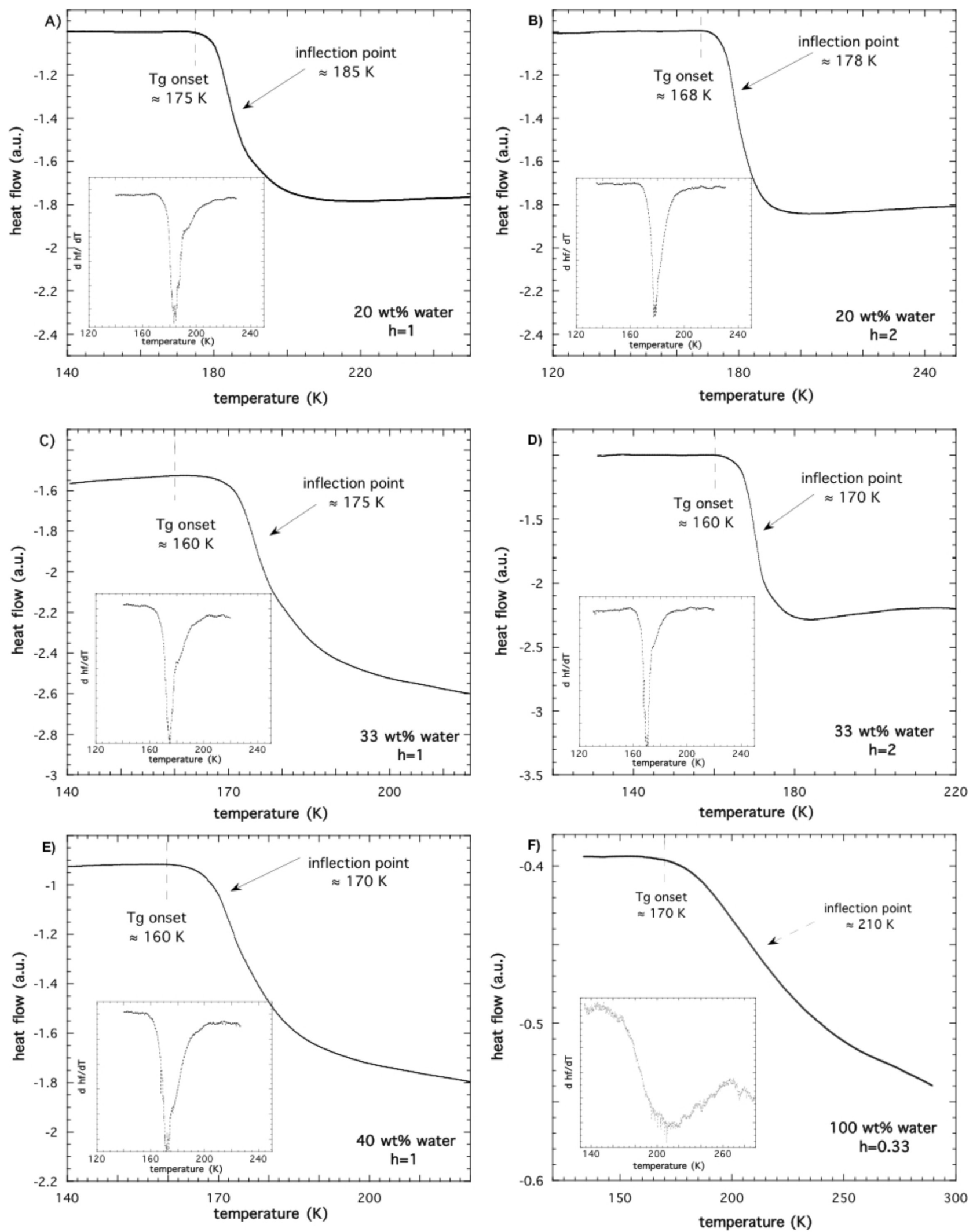

Figure 1. DSC curves obtained for myoglobin in water-glycerol mixtures with $20 \mathrm{wt} \%$ water at the solvent levels $h=1$ (A) and $h=2$ (B), $33 \mathrm{wt} \%$ water at $h=1$ (C) and $h=2$ (D), and $40 \mathrm{wt} \%$ at $h=1$ (E) and for myglobin at the hydration level $h=0.33$ (F). The insets show the derivative of the heat flow (hf) with respect to the temperature $(T)$, from which the broadness of the glass transition range was determined. 


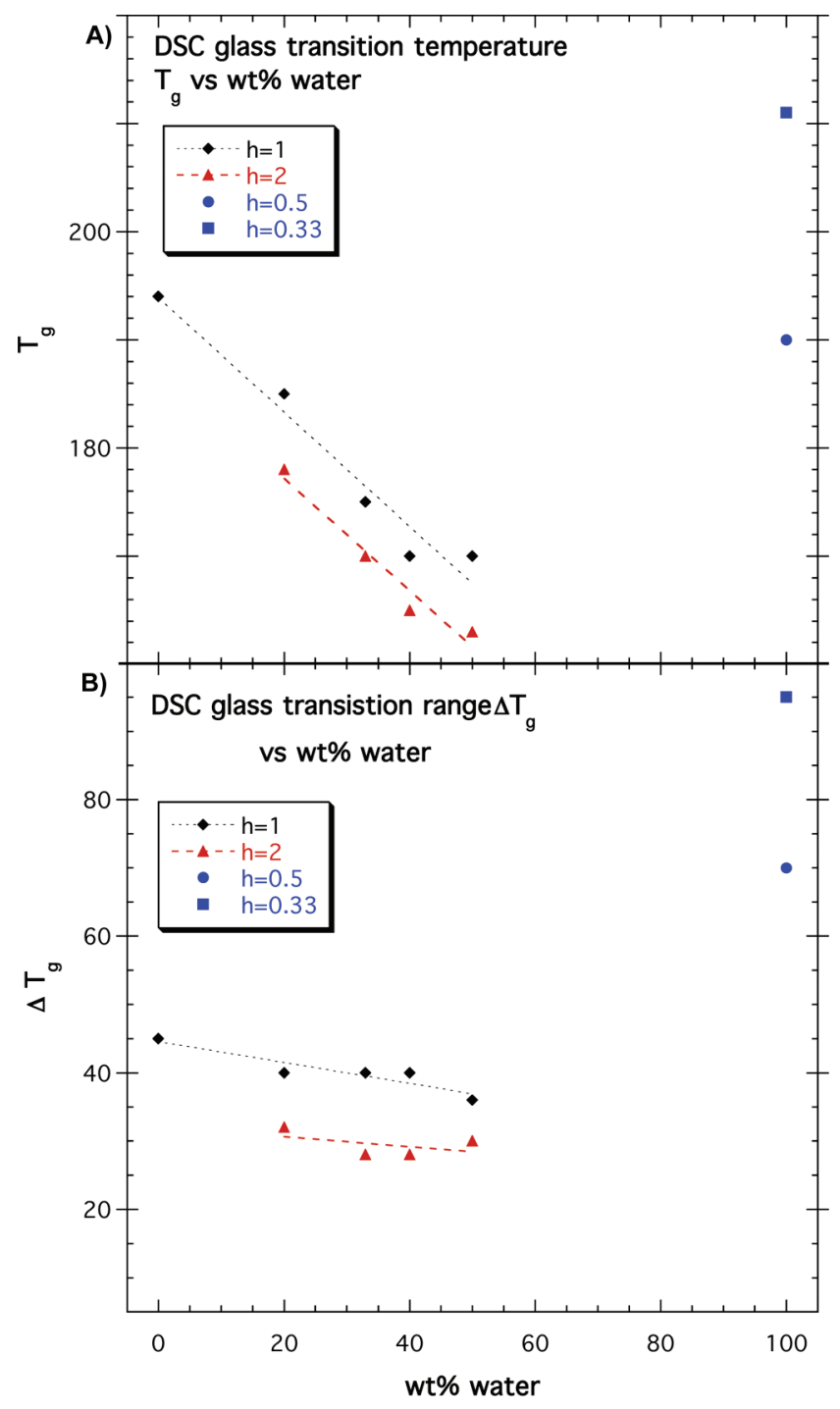

Figure 2. (A) The DSC glass transition temperature $T_{\mathrm{g}}$ (determined by the inflection point) and (B) the DSC glass transition range $\Delta T_{\mathrm{g}}$ (the whole transition range estimated from the derivative of the heat flow with respect to the temperature) shown as a function of wt $\%$ water in the solvent for all measured samples. The error in $\Delta T_{\mathrm{g}}$ is $\pm 5 \mathrm{~K}$.

For low water fractions (i.e., $20 \mathrm{wt} \%$ ), the main solvent process (process IIa in Figure 5) is, as mentioned above, due to the $\alpha$-relaxation of the solvent in the entire temperature range. However, at higher water fractions, there is a pronounced crossover from a high temperature non-Arrhenius behavior (process IIa) to a low temperature Arrhenius dependence (process IIb) at about $170 \mathrm{~K}$ in a similar way as observed for hydrated proteins (Figure 5F and ref 18 ) when the local $\beta$-relaxation of the water decouples from the structural $\alpha$-relaxation of the solvent, as discussed above. The exact time scale and activation energy of this low temperature water relaxation depend on the nature of the local environment of the relaxing water molecules (e.g., the number of hydrogen bonds to surrounding water molecules), and thereby also on the fraction of water in the sample, as shown for aqueous solutions. ${ }^{36}$ This also implies that the actual crossover temperature depends on the concentration of water in the solvent.

\section{DISCUSSION}

For hydrated myoglobin, the low temperature water process $\mathrm{IIb}$ in Figure 5F reaches a relaxation time of $100 \mathrm{~s}$ at about $125 \mathrm{~K}$. Obviously, this temperature is far below the onset temperature of the broad $T_{\mathrm{g}}$ range, which from the DSC measurements is determined to occur from $170 \mathrm{~K}$ to approximately $265 \mathrm{~K}$ (see Figure $1 \mathrm{~F}$ ). Since a secondary process is not expected to participate in a glass transition, this observation further supports the viewpoint that this process is due to a secondary $\beta$-relaxation of the hydration water below the dynamic crossover temperature, as discussed above. The calorimetric glass transition $T_{\mathrm{g}}$ of this sample occurs at about $210 \mathrm{~K}$ (see Figure $1 \mathrm{~F}$ ), which is a considerably higher temperature than the temperature of about $165 \mathrm{~K}$ where the only clearly observed protein process (process III in Figure 5F) reaches a relaxation time of $100 \mathrm{~s}$. This implies that also other slower protein processes must contribute to the calorimetrically observed glass transition of this sample, although these are too weak to be observed in the dielectric measurement. This is, however, not the case for myoglobin in water-glycerol solvents (Figure 5) and myoglobin at a higher hydration level ${ }^{16}$ where one or more additional slower protein processes can be dielectrically observed. The origin of these processes are not fully established, but one of them (process IV in Figures 5 and 6C) is in agreement, regarding both relaxation times and temperature dependence, with conformational changes of the protein structure as determined by hole-burning spectroscopy, as shown in Figure 6C. ${ }^{41}$ Although it should be noted that this process is observed on approximately the same time scale as both a slow Debye-like process and a Teflon induced "conductivity peak" recently revealed ${ }^{42}$ to occur in the corresponding pure solvents, it is clear from Figure 4 that the process cannot be a Teflon induced "conductivity peak" as it is shown also in the measurement where no Teflon film was used. Neither is it likely that its main contribution comes from the solvent, since the Debye-like process in the corresponding pure solvents is narrower and of lower dielectric strength. Therefore, we believe that process IV shown in Figures $5 \mathrm{~A}-\mathrm{E}$ and $6 \mathrm{C}$ should mainly be due to conformational changes of the protein. Further support that processes III, IV, and V in Figure 5 are due to protein motions comes from the fact that the glass transition ranges (see Figures 1 and 5) extend to considerably higher temperatures than the temperatures where the $\alpha$-relaxation in the solvents reach a relaxation time of $100 \mathrm{~s}$, i.e., freezes in on the time scale of the calorimetric measurements. Thus, glass transition related solvent motions are too fast in the high temperature range of the broad $T_{\mathrm{g}}$ range to contribute to the calorimetric $T_{\mathrm{g}}$ in that temperature range. Instead, it must be protein motions that freeze-in in the high temperature range of $T_{g}$, which, hence, supports our interpretation that processes III, IV, and V in Figure 5 are caused by glass transition related protein relaxations. In addition, by comparing results from TDDS and nuclear magnetic resonance (NMR), it has been shown that protein motions give rise to relaxations on a time scale similar to the here observed process III, as shown in Figure $6 \mathrm{~B}^{33}$

In the case of the hydrated protein, the crossover in the water dynamics (from process IIa to process IIb) observed at about 165 $\mathrm{K}$ (Figure 5F and ref 16) is close to the onset temperature of the glass transition (see Figure $1 \mathrm{~F}$ ). This is most likely not a coincidence, since the $\alpha$-like relaxation of the hydration water vanishes at the crossover temperature, ${ }^{18}$ and no glass transition related conformational changes of the protein can occur without 

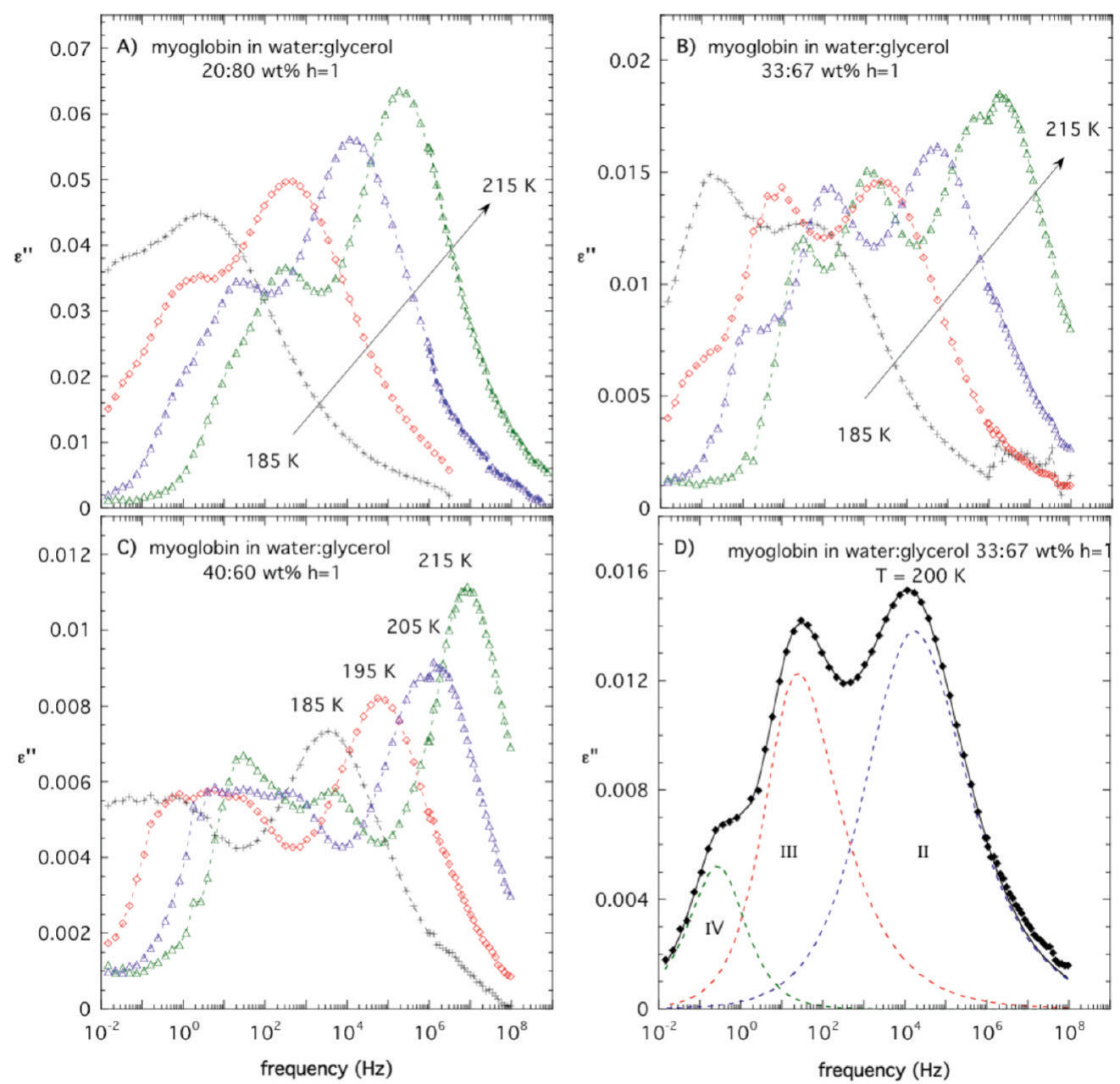

Figure 3. Temperature evolution of the imaginary part of the dielectric permittivity vs frequency for some measured myoglobin samples with total solvent contents $h$ and solvent compositions as given in the figures. For each measurement, an insulating layer of Teflon was placed between the sample and one of the electrodes. In part D, the curve fitting is shown to visualize how the relaxation times of the different relaxation processes were extracted from the measured data.

such large-scale motions in the solvent. ${ }^{4}$ Thus, the crossover in the water dynamics, and the associated vanishing of the more large-scale $\alpha$-like relaxation, seems to cause the glass transition of the protein-solvent system (see ref 16). However, probably due to the vanishing of the $\alpha$-like relaxation before the characteristic time scale of the calorimetric glass transition is reached, the interfacial water does not exhibit any clearly observable glass transition.

Our present results can be compared with several studies on different hydrated proteins, ${ }^{37,38}$ including our previous studies on hydrated myoglobin at different hydration levels. ${ }^{16,18}$ They all show a similar temperature behavior with a distinct crossover in temperature dependence from a high temperature VFT temperature dependence (process IIa) to a low temperature Arrhenius behavior (process IIb) for the water relaxation, even if it is somewhat slower in the sample at the highest hydration level $h=0.8$ (compared to the here observed process II) due to partly ice formation. ${ }^{18}$ A similar crossover in temperature dependence as observed for the water relaxation was also found for the fastest protein process in the samples of the higher hydration levels $h=$ 0.5 and $h=0.8^{16,18}$ but not in the sample at the hydration level $h=$ 0.33 in the present study. Thus, at the higher hydration levels, we found indications of a dynamic crossover to more local protein dynamics in a similar way as for the hydration water. Why no such crossover in the protein dynamics can be observed at $h=0.33$ is still unclear.

The result obtained for the water relaxation (in this study as well as in the majority of available studies) $)^{16,18,29,37,38}$ is in contrast to the result obtained in ref 48 where it was found that the water process could be described by a VFT temperature behavior in the entire measured temperature range, i.e., from high temperatures down to $T_{\mathrm{g}}$. We cannot find any reason for this inconsistency but only conclude that our present result is in accordance with other studies on water confined in a variety of crystalline host materials as well as by surfaces of biological materials, as shown by both dielectric spectroscopy and nuclear magnetic resonance (NMR). ${ }^{16,18,29,34,37,38,43-47}$ In addition, both the protein and water relaxations in ref 48 were found to be slower than the corresponding processes obtained in the present study. A possible explanation for the difference in time scales of protein motions as well as water dynamics could be the insulator material used in the present study and in ref 48 to reduce the low frequency dispersion due to conductivity and electrode polarization. We have used Teflon, which has previously been shown ${ }^{49}$ to shift relaxation peaks to slightly higher frequencies, although these shifts are very small for the present 


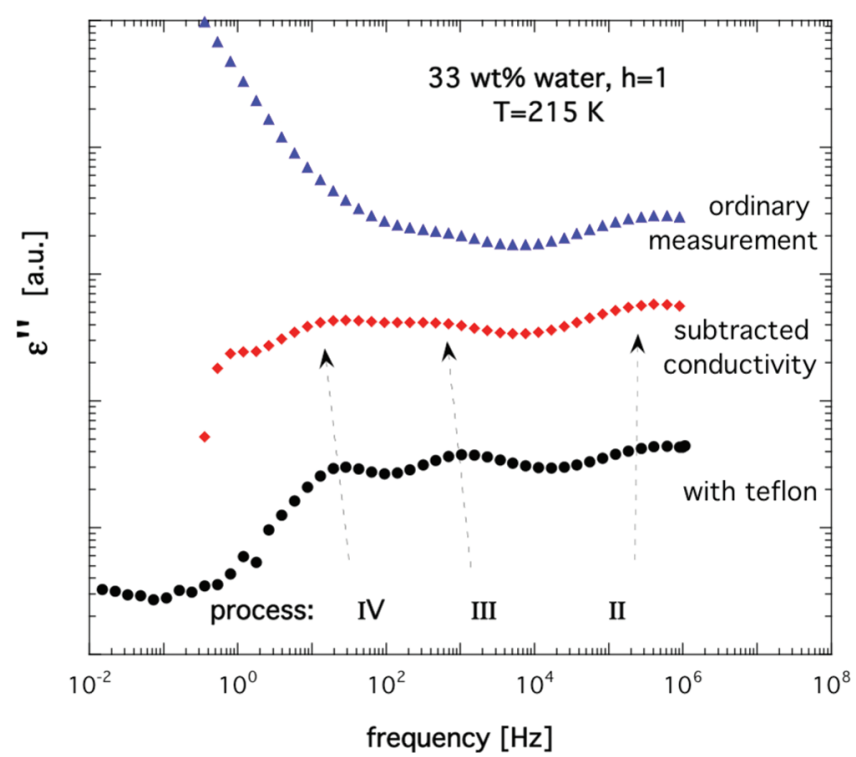

Figure 4. Comparison of experimental data on myoglobin in a waterglycerol solvent of $33 \mathrm{wt} \%$ water taken at $T=215 \mathrm{~K}$ with and without the Teflon setup. The ordinary data, shown as the imaginary part of the permittivity, taken without the Teflon setup are shown both as raw data and with the contribution from dc-conductivity subtracted. It should be noted that the subtracted data without using the Teflon setup are very similar to the data taken with the Teflon setup.

samples, as shown in Figure 4. In ref 48, Mylar was used, which may cause different shifts. Moreover, it was from that study ${ }^{48}$ concluded that the main contribution to the calorimetric protein glass transition comes from the hydration water, since there was no sign of a $T_{\mathrm{g}}$ for the dry myoglobin powder. However, the present study, in combination with many other calorimetric studies of interfacial water, ${ }^{29,47,50}$ shows clearly that interfacial water does not exhibit any observable glass transition in an ordinary calorimetric measurement. It is difficult to understand this fact, but probably motions related to the glass transition (i.e., the $\alpha$-relaxation) vanish before the glass transition temperature is reached, as also suggested from the dielectric relaxation measurements where no $\alpha$-relaxation can be observed for the hydration water below the dynamic crossover at $165 \mathrm{~K}$ (see Figure 5F). Furthermore, the glass transition range of hydrated myoglobin, as also shown in Figure $5 \mathrm{~F}$, is located at too high temperatures and is too broad to be associated with a glass transition of the hydration water, since an extrapolation of process IIa to a relaxation time of $100 \mathrm{~s}$ gives a dynamic glass transition temperature of about $147 \mathrm{~K}$. Thus, even if the $\alpha$-relaxation of the hydration water (process IIa) would have continued below the crossover temperature at $165 \mathrm{~K}$, it would not have been possible to associate it with the observed calorimetric glass transition. The same conclusion was obtained by Shinyashiki et al. ${ }^{51}$ in a dielectric study of the protein bovine serum albumin in water. Thus, as expected, protein hydration water behaves calorimetrically as water confined in other systems. $^{29,47,50}$ The observation that also dry myoglobin powder lacks a calorimetric $T_{\mathrm{g}}$ then implies that motions in the surrounding water give rise to the protein motions that freeze-in at $T_{\mathrm{g}}$. Hence, without a surrounding solvent, there are no protein motions, as previously found ${ }^{4-8}$ and also shown in this study, where the crossover in the temperature dependence of process IIa to IIb in
Figure 5F initiates the glass transition of the protein-water system. At the crossover temperature, the more cooperative $\alpha$ like relaxation (process IIa) vanishes, which, in turn, causes a freezing-in of the large-scale protein motions that give rise to the calorimetric glass transition. Thus, even if the hydration water is responsible for the glass transition by causing the protein motions, it does not give a direct relaxational contribution to the observed calorimetric glass transition, in agreement with other calorimetric studies of interfacial water. ${ }^{29,47,50}$

For myoglobin in water-glycerol mixtures of lower water fractions (less than $40 \mathrm{wt} \%$ water), a somewhat different glass transition scenario can be observed. In Figure 5A-D, it is shown that the $\alpha$-relaxation in the solvent (process IIa) reaches a relaxation time of $100 \mathrm{~s}$ (i.e., a dynamical glass transition) at about the same temperature as the onset of the calorimetric glass transition is found. For instance, in the case of $20 \mathrm{wt} \%$ water in the solvent, its relaxation time is $100 \mathrm{~s}$ at 175 and $169 \mathrm{~K}$ for the hydration levels $h=1$ and $h=2$, respectively. These temperatures are in good agreement with the onset temperatures of the calorimetric $T_{\mathrm{g}}$ at 175 and $168 \mathrm{~K}$, respectively. The fact that these glycerol containing solvents give a direct contribution to the calorimetric $T_{\mathrm{g}}$, in contrast to hydration water as discussed above, is fully consistent with the observation that the corresponding bulk solvents exhibit calorimetric $T_{\mathrm{g}}$ with similar onset temperatures. By comparing the dielectric and calorimetric results, it can, furthermore, be observed that the calorimetric $T_{\mathrm{g}}$ (given by the inflection point in the DSC thermogram) corresponds well to the temperature where the slower dielectric protein processes reach a relaxation time of $100 \mathrm{~s}$. Thus, in the cases when the solvent contains glycerol, the $\alpha$-relaxation in the solvent is present at $T_{\mathrm{g}}$ and the whole $T_{\mathrm{g}}$ range of a sample involves the freezing-in of both the $\alpha$-relaxation in the solvent as well as different types of protein fluctuations. When these protein fluctuations occur on widely different time scales, the $T_{\mathrm{g}}$ range becomes particularly broad, in accordance with previous studies of the protein glass transition. ${ }^{12,14}$

By comparing the glass transition temperatures obtained by DSC for myoglobin in the water-glycerol mixtures (Figure 1A-E) with the corresponding data on hydrated myoglobin in Figure $1 \mathrm{~F}$ and ref 16 , it is evident that the inflection point is considerably closer to the lower end of the $T_{\mathrm{g}}$ range for the glycerol containing samples. In fact, for several of the glycerol containing samples, it can be seen that the glass transition is asymmetric and seems to contain at least two components, one sharp at the lower part of the glass transition range and one broad at a slightly higher temperature, as seen in the insets of Figure 1. This is not the case for the hydrated protein, where only one broad component can be observed (see Figure 1F and ref 16). This suggests that the freezing-in of the cooperative and viscosity related $\alpha$-relaxation in the water-glycerol solvent makes a major contribution to the calorimetric $T_{\mathrm{g}}$ of these samples, in contrast to the glass transition of hydrated myoglobin where the main contribution, as discussed above, arises from protein motions occurring on different time scales. ${ }^{16}$ The fact that the solvent makes a direct contribution to the calorimetric glass transition only for myoglobin in water-glycerol mixtures, and not for hydrated myoglobin, is also evident from the much larger change in heat capacity for the glycerol containing samples during the transition; see Figure 1.

The relaxation times shown in Figure 5 indicate that glass transition related protein motions are only present at temperatures above the glass transition of the solvent, or the dynamic 

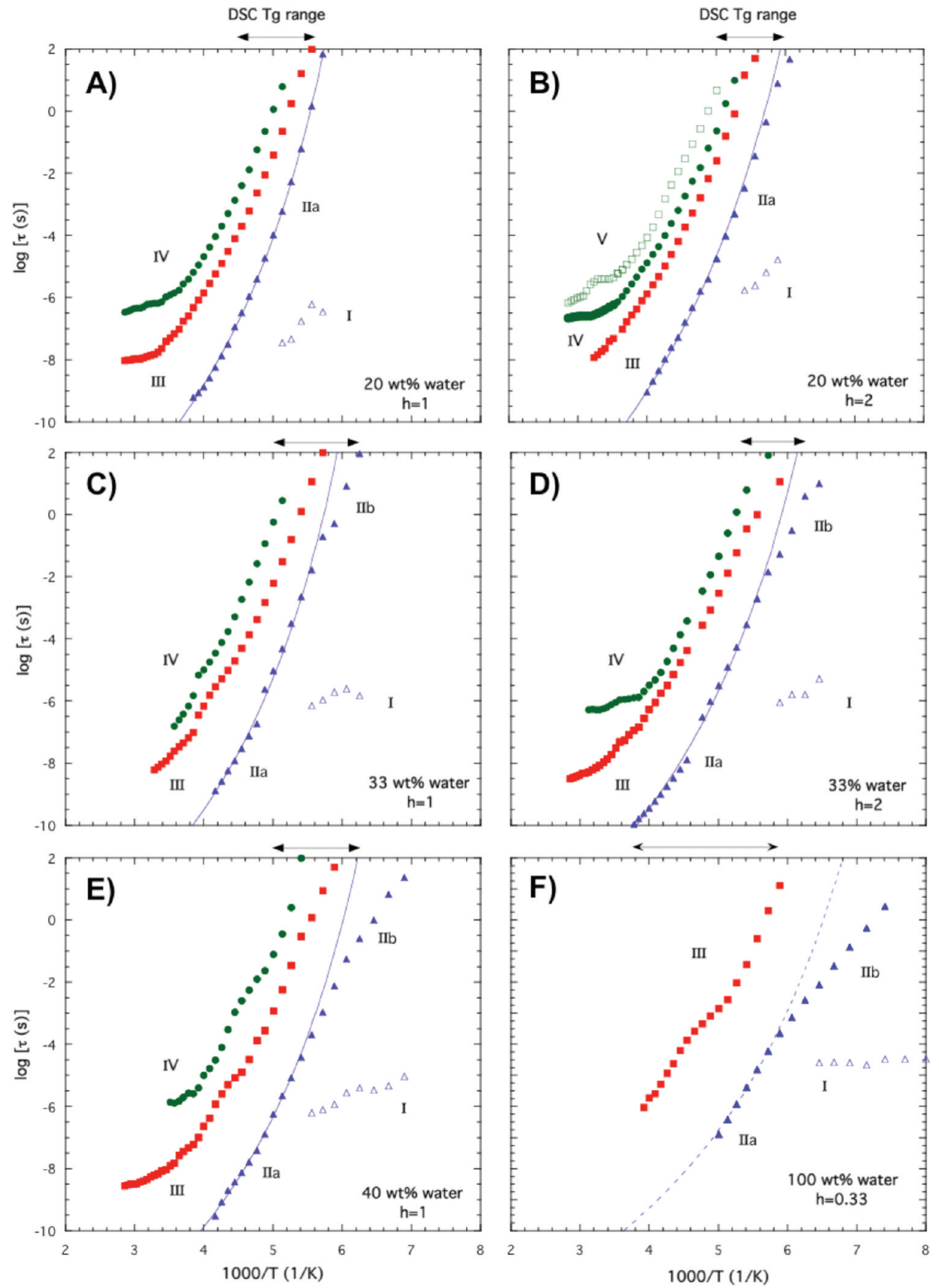

Figure 5. Dielectric relaxation times for six myoglobin samples with total solvent contents and water fractions (given in wt \% of the total solvent) as given in the figure. Also shown in the figure are the DSC glass transition ranges, $\Delta T_{\mathrm{g}}$, obtained in Figure 1 . The lines show the results of VFT (eq 3 ) curve fitting of the temperature dependence of the main solvent relaxation in the high temperature range, that is, process IIa.

crossover temperature in the case of hydration water. ${ }^{16}$ It is also clear that the protein processes exhibit similar temperature dependences as the $\alpha$-relaxation of the solvent. This is an interesting observation, since, according to Fenimore et al., ${ }^{4}$ 


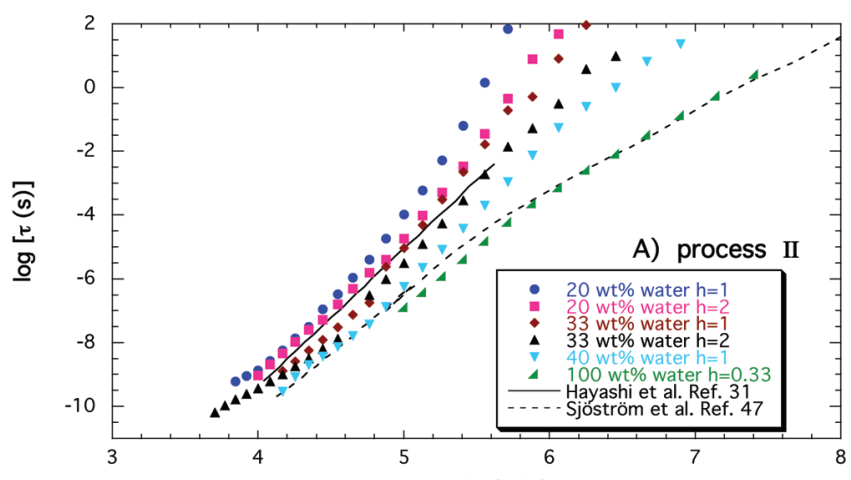

$1000 / \mathrm{T}(1 / \mathrm{K})$
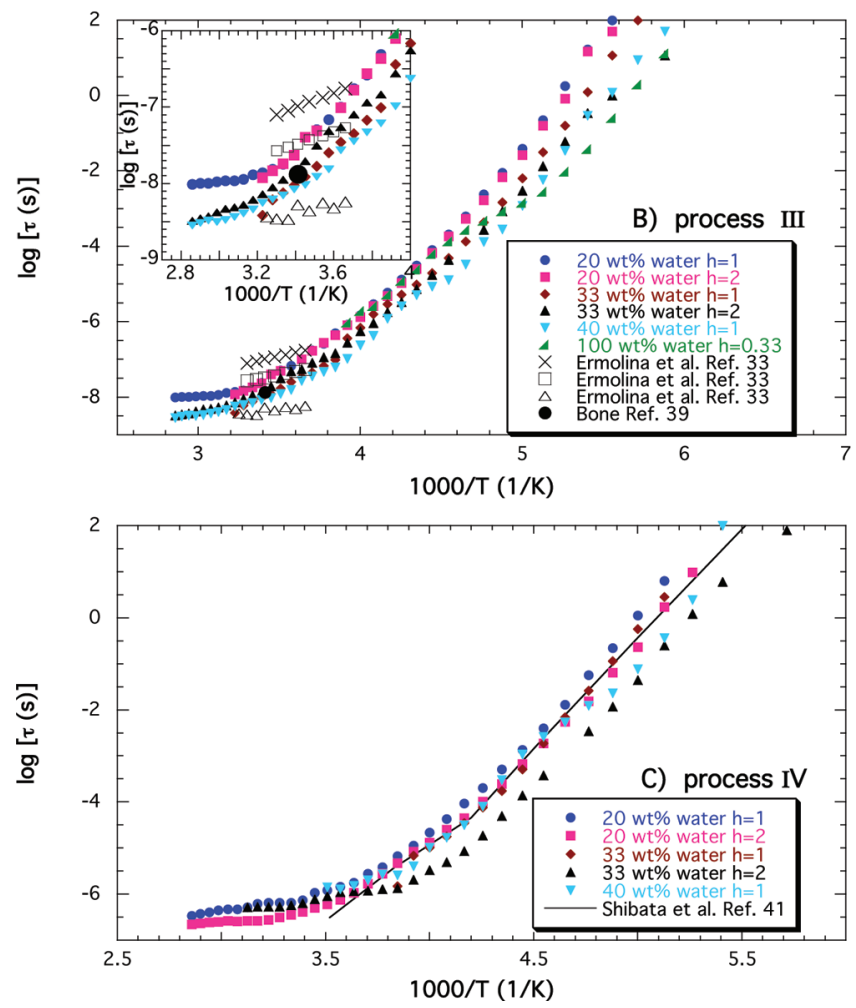

Figure 6. Dielectric relaxation times for the three main processes in the myoglobin samples, together with data from the literature as indicated in the legends of the figure. In part A, the main solvent relaxation is compared with results for a glycerol-water mixture of about 31 wt \% water $^{31}$ and the main water relaxation found in MCM- $41 .{ }^{47}$ Part B shows the fastest of the obtained protein relaxations (process III), which is attributed to the motion of polar groups on the protein surface, in accordance with the interpretation made in ref 39. In addition, results from a combined NMR and TDDS study ${ }^{33}$ on protein motions are also included in the figure. In part C, process IV is shown together with data on conformational changes of the protein molecule as obtained by holeburning spectroscopy. ${ }^{41}$

the motions of a protein can be considered as "slaved" or driven by the surrounding solvent. In their model for protein dynamics, more local solvent motions influence local internal protein motions, whereas large-scale protein fluctuations need global viscosity related solvent motions. However, this does not mean that a solvent relaxation and its associated protein motions occur on the same time scale (i.e., having the same relaxation rate); rather, it only implies that the relaxation times of the processes show similar temperature dependences, i.e., similar activation energies at a given temperature. The reason that the protein motions are generally slower than their related solvent motions is that a conformational change of a protein often requires a large number of elementary steps, which can only take place if the solvent moves. For a conceptual understanding of the different time scales, an analogy to Brownian motion of macromolecules in a solution can be made.

In order to elucidate the relation between solvent and protein dynamics in more detail, we show in Figure 7 the relaxation times of the protein processes as a function of the relaxation time for the $\alpha$-process in the solvent (process IIa in Figure 5). From the figure, it is evident that there are linear dependences (i.e., slopes of unity in the log-log plots) for all the shown protein processes and sample compositions, except maybe for the hydrated sample (Figure 7F) where the processes can be compared only in a limited temperature interval $(170-200 \mathrm{~K})$. Thus, in general, we observe the expected behavior for solvent-slaved protein processes. $^{3,4,8}$ Therefore, our findings support the study by Fenimore et al., ${ }^{3}$ where it was shown that the more global conformational changes of a protein are slaved by the $\alpha$-relaxation in the surrounding solvent.

Since large-scale conformational protein fluctuations are required for full biological activity of proteins, ${ }^{52}$ an important consequence of the present findings is that proteins must be almost inactive in water rich solvents at low temperatures where no $\alpha$-relaxation is present in the solvent. Thus, for instance, cryopreservation of protein rich food does not only slow down the biologically most important protein processes, it even prevents them from occurring if the temperature is reduced below the crossover temperature. The behavior is in close analogy with normal glass-forming liquids for which the time scale of the cooperative $\alpha$-relaxation basically goes to infinity and only local $\beta$-relaxations remain below the glass transition temperature. However, as shown in this paper, the protein does not undergo any glass transition without help from its surrounding solvent. Hence, in contrast to ordinary glasses, the glass transition is not an intrinsic property of proteins. Instead, the glass transition and other properties of proteins are driven by motions in the solvent, although the conformational changes of a protein generally occur on substantially longer time scales than the solvent relaxation. In Figure 7 , it is seen that the relaxation rates of the protein processes are typically $10^{3}-10^{6}$ times slower than the $\alpha$-relaxation in the solvent, proving the complex nature of global protein fluctuations and the associated energy landscape.

\section{CONCLUSIONS}

In this study, we show that the glass transition of myoglobin in water-glycerol solvent mixtures is strongly dependent on the fraction of water in the solvent as well as the total amount of solvent. The glass transition temperature decreases substantially both with increasing water fraction and increasing total amount of solvent. Furthermore, the broadness of the glass transition increases with decreasing solvent content. These effects are partly caused by changes in the solvent dynamics (e.g., due to the reducing viscosity with increasing water content and the increasing confinement effects with decreasing total solvent content), but also alterations in the protein dynamics must contribute, particularly to changes in the broadness of the glass transition. Therefore, we believe that the reason for the differences in the broadness of the glass transition is that the calorimetric glass transition involves several different types of 

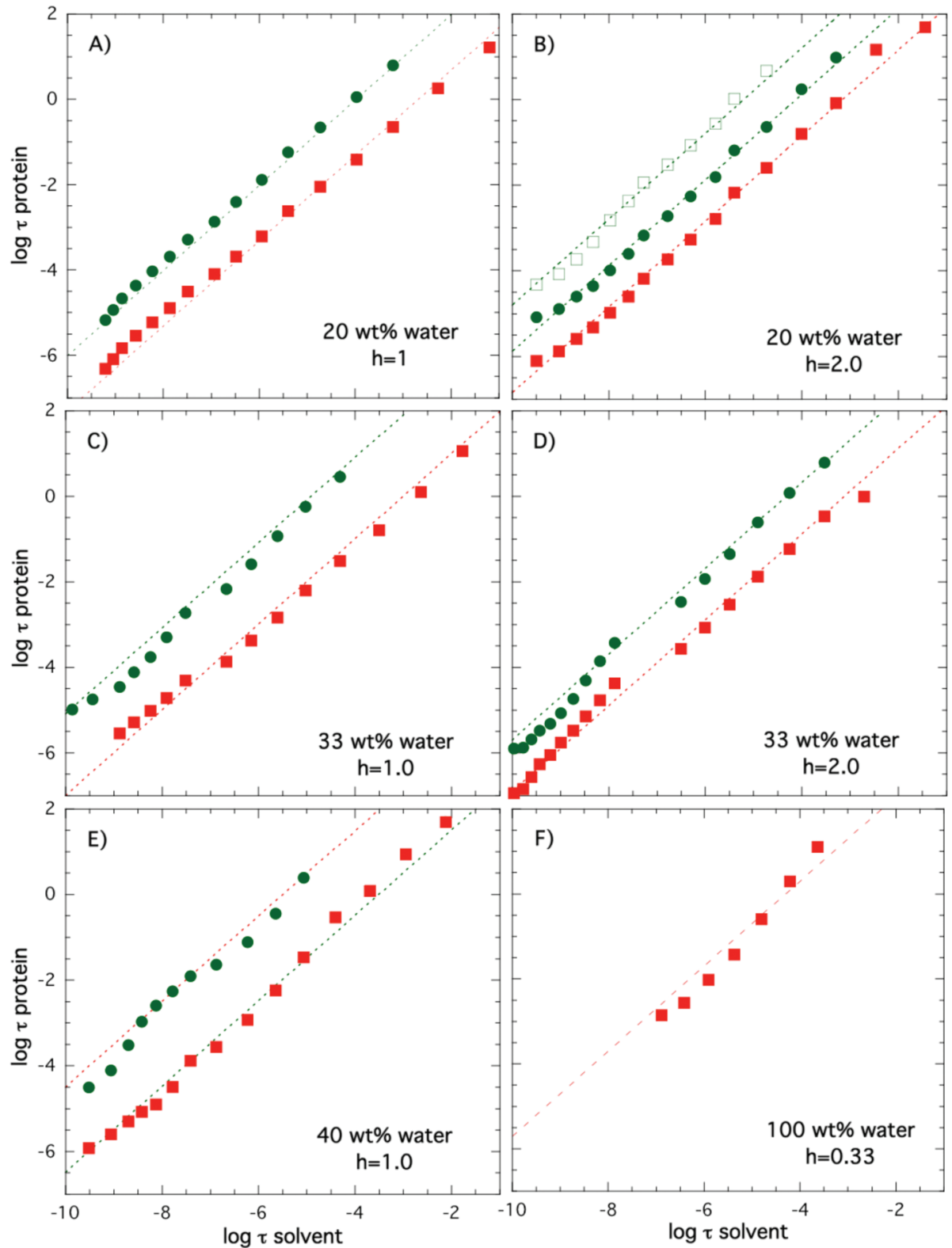

Figure 7. The relaxation times of the protein processes shown as a function of the relaxation time for the $\alpha$-process in the solvent. The protein processes are the same (same symbols), as shown in Figure 5. Note the almost perfect linear dependences for all protein processes and samples, except for the hydrated sample (F) where the processes can be compared only in a limited temperature range.

relaxation processes occurring on different time scales, and the difference in relaxation time between these processes increases with decreasing solvent content.

We also show that the origin of the calorimetric glass transition is distinctly different for myoglobin in a water-glycerol solvent compared to hydrated myoglobin. For the glycerol containing samples in this study, the viscosity related and cooperative $\alpha$ relaxation in the solvent makes a major contribution to the protein glass transition. In contrast, for hydrated myoglobin, the main contribution to the calorimetric glass transition is the freezing-in of different protein motions. ${ }^{16}$ In this case, the surrounding water does not directly contribute to the calorimetric $T_{\mathrm{g}}$ step, although the hydration water is still responsible for the protein motions that freeze-in at $T_{\mathrm{g}}$. The reason for this difference between the glycerol containing samples and the hydrated samples is most likely that for the glycerol rich samples both protein and solvent dynamics seem to be of global character in the temperature range where these processes reach the relaxation time of the calorimetric glass transition, while in the case of hydrated myoglobin the hydration water exhibits a dynamic crossover at $180 \pm 20 \mathrm{~K}$ to a local $\beta$ relaxation at low temperatures. This local water relaxation does not contribute to the calorimetric glass transition, and when the high temperature $\alpha$-like relaxation in the hydration water transforms to this local process, also the cooperative protein motions disappear. An explanation for this could be that the local $\beta$-relaxation in the solvent can only promote local noncooperative protein motions, which means that the more global protein motions disappear at the same temperature as more large-scale motions in the solvent vanish. Thus, the present findings support the conclusion by Frauenfelder and co-workers ${ }^{4,8}$ that large scale conformational 
changes of the protein are determined by the $\alpha$-relaxation in the solvent and more local protein motions are caused by the $\beta$ relaxation in the hydration shell, although, from this study, we have no direct evidence that the $\beta$-relaxation in the solvent (or hydration shell) causes local protein motions.

An important implication of our results is that cryopreservation of protein rich food should be very effective if the temperature is lowered below the crossover temperature.

\section{AUTHOR INFORMATION}

\section{Corresponding Author}

*E-mail: jan.swenson@chalmers.se.

\section{ACKNOWLEDGMENT}

We thank Prof. Hans Frauenfelder for valuable discussions. This work was financially supported by the Swedish Research Council and the Swedish Energy Agency.

\section{REFERENCES}

(1) Rupley, J. A.; Gratton, E.; Careri, G. Trends Biochem. Sci. 1983, 8,18

(2) Frauenfelder, H.; Gratton, E. Methods Enzymol. 1986, 127, 207.

(3) Fenimore, P. W.; Frauenfelder, H.; McMahon, B. H.; Parak, F. G. Proc. Natl. Acad. Sci. U.S.A. 2002, 99, 16047.

(4) Fenimore, P. W.; Frauenfelder, H.; McMahon, B. H.; Young,

R. D. Proc. Natl. Acad. Sci. U.S.A. 2004, 101, 14408.

(5) Tarek, M.; Tobias, D. J. Phys. Rev. Lett. 2002, 88, 1381011.

(6) Vitkup, D.; Ringe, D.; Petsko, G. A.; Karplus, M. Nat. Struct. Biol. 2000, 7, 34 .

(7) Doster, W.; Settles, M. Biochim. Biophys. Acta, Proteins Proteomics 2005, 1749, 173.

(8) Frauenfelder, H.; Chen, G.; Berendzen, J.; Fenimore, P. W.; Jansson, H.; McMahon, B. H.; Mihut-Stroe, I.; Swenson, J.; Young, R. D. Proc. Natl. Acad. Sci. U.S.A. 2009, 106, 5129.

(9) Frauenfelder, H.; Sligar, S. G.; Wolynes, P. G. Science 1991, 254, 1598.

(10) Angell, C. A. Science 1995, 267, 1924.

(11) Sartor, G.; Hallbrucker, A.; Hofer, K.; Mayer, E. J. Phys. Chem. 1992, 96, 5133.

(12) Sartor, G.; Mayer, E.; Johari, G. P. Biophys. J. 1994, 66, 249.

(13) Brownsey, G. J.; Noel, T. R.; Parker, R; Ring, S. G. Biophys. J. 2003, 85, 3943.

(14) Miyazaki, Y.; Matsuo, T.; Suga, H. J. Phys. Chem. B 2000, 104, 8044.

(15) Doster, W.; Bachleitner, A.; Dunau, R.; Hiebl, M.; Luscher, E. Biophys. J. 1986, 50, 213.

(16) Jansson, H.; Swenson, J. Biochim. Biophys. Acta, Proteins Proteomics 2010, 1804, 20.

(17) Rariy, R. V.; Klibanov, A. M. Proc. Natl. Acad. Sci. U.S.A. 1997, 94, 13520 .

(18) Swenson, J.; Jansson, H.; Bergman, R. Phys. Rev. Lett. 2006, 96, 2478021.

(19) Morimoto, T.; Iwaki, T. J. Chem. Soc., Faraday Trans. 1 1987, $83,943$.

(20) Costa, M. E. V.; Mantas, P. Q.; Baptista, J. L. Sens. Actuators, B $1995,27,312$.

(21) Richert, R. Eur. Phys. J. B 2009, 68, 197.

(22) Wubbenhorst, M.; van Turnhout, J. J. Non-Cryst. Solids 2002, 305,40 .

(23) Havriliak, S.; Negami, S. Polymer 1967, 8, 161.

(24) Bergman, R. J. Appl. Phys. 2000, 88, 1356.

(25) Vogel, H. Phys. Z. 1921, 22.

(26) Fulcher, G. S. J. Am. Ceram. Soc. 1925, 8, 789.

(27) Tammann, G.; Hesse, G. Z. Anorg. Allg. Chem. 1926, 156, 245.
(28) Jansson, H.; Kargl, F.; Fernandez-Alonso, F.; Swenson, J. J. Chem. Phys. 2009, 130, 205101.

(29) Swenson, J.; Jansson, H.; Hedström, J.; Bergman, R. J. Phys.: Condens. Matter 2007, 19, 205109.

(30) Puzenko, A.; Hayashi, Y.; Ryabov, Y. E.; Balin, I.; Feldman, Y.; Kaatze, U.; Behrends, R. J. Phys. Chem. B 2005, 109, 6031.

(31) Hayashi, Y.; Puzenko, A.; Balin, I.; Ryabov, Y. E.; Feldman, Y. J. Phys. Chem. B 2005, 109, 9174.

(32) Khodadadi, S.; Pawlus, S.; Roh, J. H.; Sakai, V. G.; Mamontov, E.; Sokolov, A. P. J. Chem. Phys. 2008, 128, 195106.

(33) Ermolina, I.; Fedotov, V.; Feldman, Y.; Ivoylov, I. J. Non-Cryst. Solids 1994, 172, 1103.

(34) Cerveny, S.; Schwartz, G. A.; Bergman, R.; Swenson, J. Phys. Rev. Lett. 2004, 93, 245702.

(35) Capaccioli, S.; Ngai, K. L.; Shinyashiki, N. J. Phys. Chem. B 2007, $111,8197$.

(36) Sjöström, J.; Mattsson, J.; Bergman, R.; Johansson, E.; Josefsson, K; Svantesson, D.; Swenson, J. Phys. Chem. Chem. Phys. 2010, $12,10452$.

(37) Vogel, M. Phys. Rev. Lett. 2008, 101, 2257011.

(38) Lusceac, S. A.; Vogel, M. J. Phys. Chem. B 2010, 114, 10209.

(39) Bone, S. Biochim. Biophys. Acta 1987, 916, 128.

(40) Sinibaldi, R.; Ortore, M. G.; Spinozzi, F.; Carsughi, F.; Frielinghaus, H.; Cinelli, S.; Onori, G.; Mariani, P. J. Chem. Phys. 2007, 126.

(41) Shibata, Y.; Kurita, A.; Kushida, T. Biophys. J. 1998, 75, 521.

(42) Jansson, H.; Bergman, R.; Swenson, J. Phys. Rev. Lett. 2010, 104, 017802.

(43) Jansson, H.; Swenson, J. Eur. Phys. J. E 2003, 12, S51.

(44) Swenson, J.; Jansson, H.; Howells, W. S.; Longeville, S. J. Chem. Phys. 2005, 122, 084505.

(45) Jansson, H.; Bergman, R.; Swenson, J. J. Phys. Chem. B 2005, 109, 24134.

(46) Cerveny, S.; Alegria, A.; Colmenero, J. Phys. Rev. E 2008, 77.

(47) Sjöström, J.; Swenson, J.; Bergman, R.; Kittaka, S. J. Chem. Phys. 2008, 128, 154503. Hedström, J.; Swenson, J.; Bergman, R.; Jansson, H.; Kittaka, S. Eur. Phys. J. Special Topics 2007, 141, 53.

(48) Schiro, G.; Cupane, A.; Vitrano, E.; Bruni, F. J. Phys. Chem. B 2009, 113, 9606.

(49) Bergman, R.; Jansson, H.; Swenson, J. J. Chem. Phys. 2010, $132,044504$.

(50) Cerveny, S.; Barroso-Bujans, F.; Alegriá, A.; Colmenero, J. J. Phys. Chem. C 2010, 114, 2604.

(51) Shinyashiki, N.; Yamamoto, W.; Yokoyama, A.; Yoshinari, T.; Yagihara, S.; Kita, R.; Ngai, K. L.; Capaccioli, S. J. Phys. Chem. B 2009, 113, 14448.

(52) Parak, F. G. Curr. Opin. Struct. Biol. 2003, 13, 552. 